\title{
Ecological and Human Health Risk Assessment of Pesticide Residues in Fish and Sediments from Vea Irrigation Reservoir
}

\author{
Kanton Bello Richard Danladi, Osei Akoto \\ Chemistry Department, Kwame Nkrumah University of Science and Technology, Kumasi, Ghana \\ Email: kantondan@gmail.com,wofakkmann@yahoo.com
}

How to cite this paper: Danladi, K.B.R. and Akoto, O. (2021) Ecological and $\mathrm{Hu}$ man Health Risk Assessment of Pesticide Residues in Fish and Sediments from Vea Irrigation Reservoir. Journal of Environmental Protection, 12, 265-279.

https://doi.org/10.4236/jep.2021.124017

Received: February 12, 2021

Accepted: April 22, 2021

Published: April 25, 2021

Copyright $\odot 2021$ by author(s) and Scientific Research Publishing Inc. This work is licensed under the Creative Commons Attribution International License (CC BY 4.0).

http://creativecommons.org/licenses/by/4.0/

\begin{abstract}
The low yield of food production ascribed to harm caused by pests has led to the application of pesticides to food crops. Pesticide residues from the application on crops are mostly found in foods that can cause diseases for consumers of such products. A total of 37 pesticide residues consisting of 15 organochlorines (OC), 13 organophosphorus (OP) and 9 synthetic pyrethroids (SP) were determined. The QuEChERS method was exploited for extraction and clean-up. Gas Chromatograph was used for detection and quantification which was equipped with an electron capture detector and pulse flame photometric detector. The results showed that the mean concentrations of pesticides in fish ranged from $0.007 \mathrm{mg} \cdot \mathrm{kg}^{-1}$ to $1.026 \mathrm{mg} \cdot \mathrm{kg}^{-1}$ for OCs, 0.002 $\mathrm{mg} \cdot \mathrm{kg}^{-1}$ to $0.190 \mathrm{mg} \cdot \mathrm{kg}^{-1}$ for OPs and $0.004 \mathrm{mg} \cdot \mathrm{kg}^{-1}$ to $0.032 \mathrm{mg} \cdot \mathrm{kg}^{-1}$ for SP. Sediments have mean concentrations ranged from $0.005 \mathrm{mg} \cdot \mathrm{kg}^{-1}$ to 1.207 $\mathrm{mg} \cdot \mathrm{kg}^{-1}$ for OCs. OP ranges from $0.002 \mathrm{mg} \cdot \mathrm{kg}^{-1}$ to $0.399 \mathrm{mg} \cdot \mathrm{kg}^{-1}$ and 0.003 $\mathrm{mg} \cdot \mathrm{kg}^{-1}$ to $0.202 \mathrm{mg} \cdot \mathrm{kg}^{-1}$ for synthetic pyrethroids. Maximum Residue Limits were exceeded in both fish and sediment samples except for malathion, fenitrothion, profenofos, gamma-chlordane, and deltamethrin. Exposure in children ranged from $4.60 \times 10^{-6} \mathrm{mg} \cdot \mathrm{kg}^{-1} \cdot \mathrm{d}^{-1}$ to $2.36 \times 10^{-3} \mathrm{mg} \cdot \mathrm{kg}^{-1} \cdot \mathrm{d}^{-1}$ and in adults it is from $1.97 \times 10^{-6} \mathrm{mg} \cdot \mathrm{kg}^{-1} \cdot \mathrm{d}^{-1}$ to $1.01 \times 10^{-3} \mathrm{mg} \cdot \mathrm{kg}^{-1} \cdot \mathrm{d}^{-1}$. Health risk estimation revealed a non-cancer risk potential of $\beta-\mathrm{HCH}$ in sediment and aldrin and p,p'-DDE in fish. Carcinogenic risk assessed for organochlorine pesticide residues indicates cancer benchmark concentrations greater than $10^{-4}$ to $10^{-6}$ threshold for acceptance.
\end{abstract}

\section{Keywords}

Pesticides, Risk, Carcinogenic, Organochlorine, Organophosphorus, Synthetic Pyrethroids 


\section{Introduction}

Pesticides are widely used in agricultural and sanitation sectors for combating pests in Ghana [1]. The use of pesticides in agricultural lands to control pests brings about bumper harvest to farmers whiles producing toxics to non-target organisms [2] such as fish [3]. Pesticide usage in Ghana continues to increase as agricultural production escalates. This increase in pesticide usage brings with it environmental and health ills arising from indiscriminate use and inappropriate handling of the chemicals. Workers exposed to pesticide are often illiterate and lack training, no right equipment and adequate safety information. Lack of legislative controls, susceptible population and the availability of highly toxic pesticides which often are poorly labelled and badly package and irresponsibly promoted are serious factors accounting for the hazards of pesticide use in Ghana [4]. The toxic, persistent pesticides may spread within the reservoir deteriorating the quality of the water gradually jeopardizing its use for drinking by humans and livestock. Non-target flora and fauna concentrate these chemicals in their tissues and pass them on along the food chain. The accumulation of such pollutants in food chain may restrict the consumption of valuable food resource like fish [5].

Modern agriculture relies to a great extent on pesticides application to feed the ever growing large populations around the world [6]. Humans and the environment are exposed to several dangers from long and increased use of pesticides [7]. A well planned and enacted legislation and massive pressure from environmentalists is essential for the enforcement and supervision of pesticides use [8]. This will help obtain high benefits with very low risk to humans and the environment [9]. Approximately $87 \%$ of Ghanaian farmers use chemical pesticides to control pests and diseases on vegetables and fruits [10]. The risk posed by pesticide residues to humans and the environment varies with the toxicological, physical and ecological properties of the pesticide. In Ghana, fresh or processed fish is a major source of protein for both humans and animals [1]. The USA uses about 5 million $\mathrm{kg}$ of pesticides each year with agriculture accounting for $70 \%$ $80 \%$ of the total pesticide use [11].

The risk posed by pesticides to aquatic life is relative and depends on several factors including landscape, application method, application rate, erosion, irrigation, topography, soil type and land management practices [12]. Sea foods such as fish, lobsters, prawns, mussels and oysters are generally classified as a rich source of protein and other nutrients [13]. Invariably, an increase in concentrations of pesticide residues in fish and sediments, is an indication of the high level of pollution of the water throughout its food web [14].

The use OCs and many different kinds of OPs have been banned in Ghana, yet they are still in use by many farmers across the country. This is because they are still effective in the control of pests and relatively inexpensive compared to the legally allowed once [4].

The increase presence of pesticide residuals around the catchment area of the 
Vea irrigation reservoir is related to unguided and ignorance of the applicants leading to runoffs polluting the ecosystem and contaminating the organisms in the reservoir.

This research seeks to assess the pesticide residues of organochlorines (OCs), synthetic Pyrethroids (SPs) and organophosphorus (OPs) in three (3) different fish species of tilapia (Oreochromis niloticus), sardi (Alestes baremose) and African mudfish or catfish (Clarias gariepinus) and in sediment samples from the Vea irrigation reservoir.

\section{Methodology}

\subsection{Description of Study Area}

Vea is a community under the Bongo District of the Upper East Region of Ghana. The Vea reservoir is located between latitude $10^{\circ} 45^{\prime} \mathrm{N}$ and longitude $1^{\circ} \mathrm{W}$. The vegetation in the study area is dry guinea savannah, distinguished by short grasses and short trees. The climate has a mean minimum and maximum temperature of $14^{\circ} \mathrm{C}$ and $40^{\circ} \mathrm{C}$, respectively. The mean annual rainfall ranges from 850 to $1000 \mathrm{~mm}$ which occurs in the months from May to October, followed by a prolonged dry season [15]. The first part of the dry season from November to mid-February is characteristically cold and dry with dusty Harmattan winds. The rest of the dry season is usually characterized by a wide temperature range from $14^{\circ} \mathrm{C}$ at night and to over $35^{\circ} \mathrm{C}$ during the day. Humidity is also very low, making the daytime temperature high and less comfortable. The Vea irrigation project is constructed on the tributary of the White Volta River from Burkina Faso and serves eight communities with a total farmer population of 6000 as shown in Figure 1. The surface area of Vea reservoir is 405 ha with a maximum storage of $1.7 \times 10^{7} \mathrm{~m}^{3}$ serving $21 \mathrm{~km}$ of main canal distance [15].
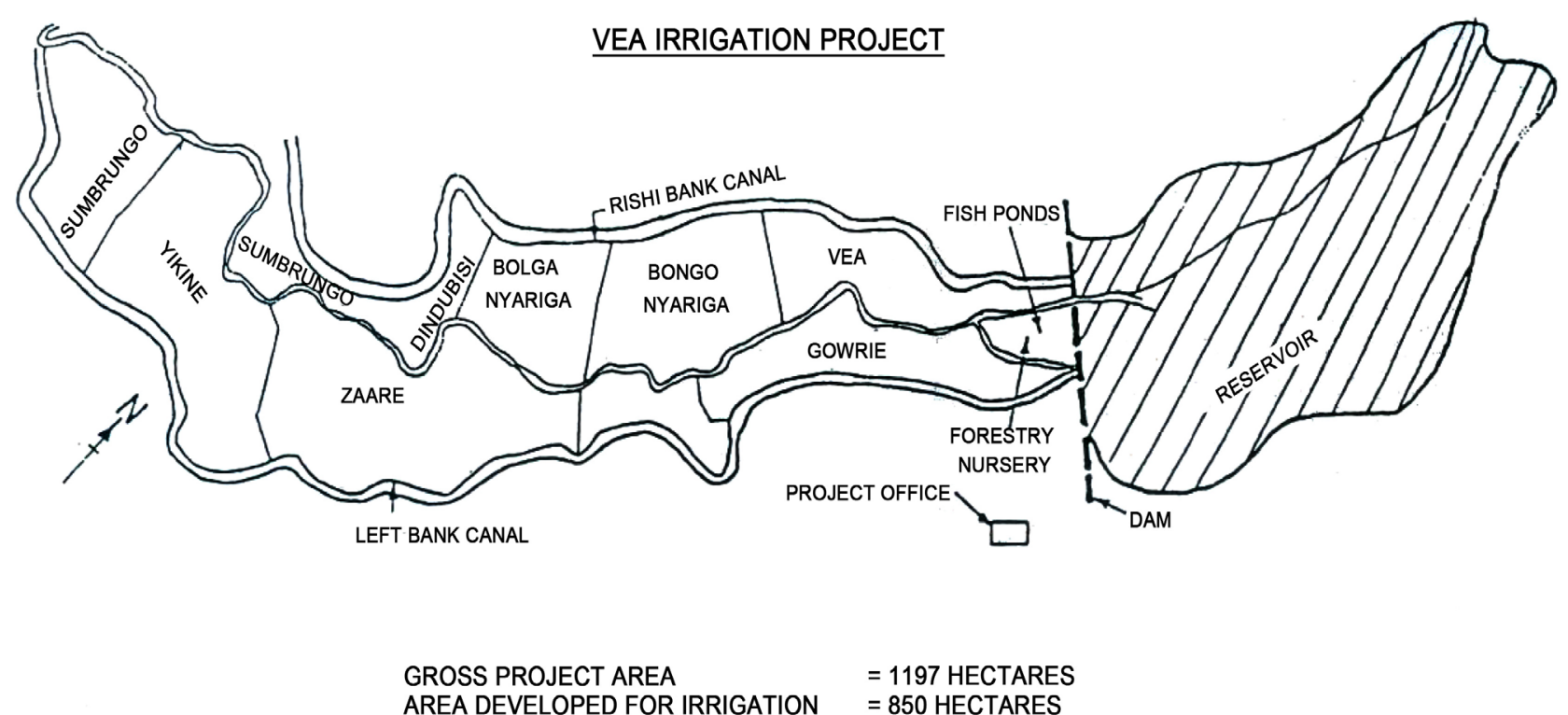

Figure 1. Map of communities within the catchment area Vea reservoir. 


\subsection{Sampling}

Three different fish species; tilapia (Oreochromis niloticus), sardi (Alestes baremose) and African catfish (Clarias gariepinus) were considered for this work, because they are the most frequently eaten in the study area. A total of seventy-five (75) fish samples, twenty-five (25) each were purchased from fishermen at the landing site of the reservoir with another twenty-five (25) sediment samples which were evenly taken from different parts of the reservoir.

The fish samples were first wrapped in aluminum foil and packed in labelled clean polyethylene bags as with the sediment samples and transported in thermos-insulated containers with ice packs to the laboratories of KNUST where they were stored at temperature below $-10^{\circ} \mathrm{C}$ until processed.

\subsection{Sample Preparation and Extraction}

The sediment samples were air dried for one week. Stalks, stones and other debris were removed and each sample homogenized to maintain homogeneity. These samples were then ground and passed through a $2 \mathrm{~mm}$ sieve to remove the coarse sediment fraction and kept in plastic bags. The fish samples were identified at the Department of Fishery of KNUST. They were thawed, cleaned with distilled water and their scales sloughed. Their muscle tissues dissected, minced into pieces and homogenized. The extraction of pesticide residues in fish and in sediments was done according to the method developed by [16] with some modifications.

Pesticide grade acetonitrile, analytical grade sodium chloride, magnesium sulphate anhydrous, ethyl acetate, pesticide standards were obtained from $\mathrm{BDH}$ Laboratory Supplies, England. Reference Standards were certified by Dr. Ehrenstorfer Laboratories (GmbH Germany) and Silica and C18 were obtained from Phenomenex, USA.

Ten (10.0) g of homogenous fish sample was weighed into a $250 \mathrm{~mL}$ nalgene jar and $50 \mathrm{~mL}$ acetonitrile was added and macerate for $2 \mathrm{~min}$. Centrifugation was done for $3 \mathrm{~min}$ at 3000 turns/min. The extract was then filtered through a filter paper into a $100 \mathrm{~mL}$ volumetric flask. Another $(20 \mathrm{~mL})$ acetonitrile was added to the residue, macerated for $1 \mathrm{~min}$ and the dispersing element rinsed with acetonitrile into the jar and centrifuged for $3 \mathrm{~min}$. at 3000 turns/min. The residue was filtered again into the $100 \mathrm{~mL}$ volumetric flask, and the jar rinsed for residue with acetonitrile and adjusted the filtrate to the $100 \mathrm{~mL}$ mark. Fifty (50) $\mathrm{mL}$ residue was transferred into a round bottom flask, and concentrated on the rotary evaporator to $2 \mathrm{~mL}$ for adsorption chromatography.

\subsection{Sample Clean-Up in Fish Extract}

A Bond Elute C-18 was conditioned with a $1000 \mathrm{mg} / 6 \mathrm{~mL}$ cartridge with $(6 \mathrm{~mL})$ acetonitrile and the sample extract were poured into the column and the column eluted with $10 \mathrm{~mL}$ acetonitrile. A Silica SPE cartridge was conditioned with 1000 $\mathrm{mg} / 6 \mathrm{~mL}$ which has $2 \mathrm{~g}$ of $\mathrm{MgSO}_{4}$ on top with $(6 \mathrm{~mL})$ acetonitrile. The extract 
was poured unto the column and eluted with $10 \mathrm{~mL}$ acetonitrile. The extract was concentrated below $40^{\circ} \mathrm{C}$ on the rotary evaporator to dryness. The filtrate was re-dissolved in $1 \mathrm{~mL}$ ethyl acetate and transferred into a $2 \mathrm{~mL}$, vial for quantitation by GC-ECD.

\subsection{Extraction of Pesticide Residues in Sediment}

Ten (10.0) $\mathrm{g}$ of homogenous sediment sample was put in a $100 \mathrm{~mL}$ separating flask and $10 \mathrm{~mL}$ acetonitrile added, corked and sonicated for $5 \mathrm{~min}$. After that, a further $10 \mathrm{~mL}$ acetonitrile was added, corked and the flask place on the horizontal mechanical shaker and set to shake continuously for $30 \mathrm{~min}$. The mixture was allowed to stand for 10 minutes to separate into layers. Ten (10) $\mathrm{mL}$ of the organic phase was pipetted into a round-bottomed flask and evaporated to $2 \mathrm{~mL}$ for extract clean-up.

\subsection{Sample Clean-Up in Sediment}

A cartridge was conditioned with silica and acetonitrile $(1000 \mathrm{mg} / 6 \mathrm{~mL})$ to have a $1 \mathrm{~cm}$ thickness layer of anhydrous magnesium sulphate on top with $(10 \mathrm{~mL})$ of acetonitrile. Two (2) $\mathrm{mL}$ of the extract was purified by loading onto the cartridge and the eluate collected into a flask. The cartridge was further eluted with $10 \mathrm{~mL}$ of acetonitrile and the filtrate concentrated below $40^{\circ} \mathrm{C}$ on the rotary evaporator. The filtrate was re-dissolved in $1 \mathrm{~mL}$ ethyl acetate and the extract transferred into a $2 \mathrm{~mL}$, standard vial prior to quantitation by GC-ECD.

\subsection{Quality Control Analysis}

The quality of analysis of pesticide residues was enhanced through solvent blanks and spikes. The solvent blanks were used to check for interferences from reagents, whilst the spike samples were used to determine recovery which is an indicator of method performance and accuracy. The triplicate and duplicate measurements of samples were used to confirm method precision. The recoveries ranged between $74 \%-120 \%$ for organochlorine pesticide residues, $70 \%$ - 94\% for organophosphorus pesticide residues and $73 \%-100 \%$ for synthetic pyrethroid pesticide residues. Fortification level of $0.05 \mathrm{mg} / \mathrm{kg}$ was chosen for OCs in sediment samples and $0.01 \mathrm{mg} / \mathrm{kg}$ for fish samples based on the limit of determination of the pesticides being analysed. A gas chromatogram, Varian CP-3800 equipped with $63 \mathrm{Ni}$ electron capture detector, CTC Analytic Combi PAL autosampler, split-splitless injector, programme pneumatic control and a computer star workstation data processor were used for all classes of pesticides.

\subsection{Estimation of Exposure to Humans and Risk}

The estimated daily exposure (Ems) of individuals to pesticide residues from each fish species was determined using Equation (1);

$$
\mathrm{Em}=\frac{\mathrm{Cm} \times \mathrm{CR}^{\circ}}{\mathrm{BW}}
$$


where $\mathrm{Cm}, \mathrm{CR}^{\circ}$ and $\mathrm{BW}$ represent concentration of chemical contaminant in the muscle portion of fish $(\mathrm{mg} / \mathrm{kg})$, mean daily consumption rate of fish $(\mathrm{kg} / \mathrm{d})$ [17] and body weight of an individual consumer $(\mathrm{kg})$ [18]. The estimated daily exposures are calculated for children and adults eating contaminated fish species of tilapia, catfish and sardi as shown in Table 1 for OPs, Table 2 for OCs and Table 3 for SPs.

Table 1. Extent of exposure of OPs pesticides residues $(\mathrm{mg} / \mathrm{kg} \cdot \mathrm{d})$ in fish for children (Em1) and adults (Em2).

\begin{tabular}{|c|c|c|c|c|c|c|}
\hline \multirow{2}{*}{ Organophosphorus } & \multicolumn{2}{|c|}{ Catfish } & \multicolumn{2}{|c|}{ Tilapia } & \multicolumn{2}{|c|}{ Sardi } \\
\hline & Em1 & $\mathrm{Em} 2$ & Em1 & $\mathrm{Em} 2$ & Em 1 & $\mathrm{Em} 2$ \\
\hline Methamidophos & $2.44 \mathrm{E} 0-4$ & $1.04 \mathrm{E}-04$ & $4.37 \mathrm{E}-04$ & $1.87 \mathrm{E}-04$ & - & - \\
\hline Ethoprophos & - & - & - & - & - & - \\
\hline Dimethoate & - & - & $5.29 \mathrm{E}-05$ & $2.27 \mathrm{E}-05$ & - & - \\
\hline Pirimiphos-methyl & $4.60 \mathrm{E}-06$ & $1.97 \mathrm{E}-06$ & - & - & - & - \\
\hline Chlorpyrifos & - & - & - & - & - & - \\
\hline Malathion & $1.38 \mathrm{E}-05$ & $5.91 \mathrm{E}-06$ & $2.30 \mathrm{E}-05$ & $9.86 \mathrm{E}-06$ & $1.15 \mathrm{E}-05$ & $4.93 \mathrm{E}-06$ \\
\hline Parathion & - & - & - & - & - & - \\
\hline Chlorfenvinphos & $1.84 \mathrm{E}-05$ & $7.89 \mathrm{E}-06$ & $2.99 \mathrm{E}-05$ & $1.28 \mathrm{E}-05$ & $1.61 \mathrm{E}-05$ & $6.90 \mathrm{E}-06$ \\
\hline Profenofos & $1.38 \mathrm{E}-05$ & $5.91 \mathrm{E}-06$ & - & - & $1.38 \mathrm{E}-05$ & $5.91 \mathrm{E}-06$ \\
\hline
\end{tabular}

Table 2. Exposure of OCs pesticides residues (mg/kg.d) in fish for children (Em1) and adults (Em2).

\begin{tabular}{|c|c|c|c|c|c|c|}
\hline \multirow{2}{*}{ Organochlorines } & \multicolumn{2}{|c|}{ Catfish } & \multicolumn{2}{|c|}{ Tilapia } & \multirow{2}{*}{$\begin{array}{l}\text { Sardi } \\
\text { Em1 }\end{array}$} & \multirow{2}{*}{$\begin{array}{l}\text { Fish } \\
\text { Em2 }\end{array}$} \\
\hline & Em1 & $\operatorname{Em} 2$ & Em1 & Em2 & & \\
\hline Beta-HCH & - & - & - & - & - & - \\
\hline Lindane & - & - & - & - & - & - \\
\hline Heptachlor & $2.07 \mathrm{E}-05$ & $8.87 \mathrm{E}-06$ & $2.76 \mathrm{E}-05$ & $1.18 \mathrm{E}-05$ & $2.76 \mathrm{E}-05$ & $1.18 \mathrm{E}-05$ \\
\hline Aldrin & $4.76 \mathrm{E}-04$ & $2.04 \mathrm{E}-04$ & $2.05 \mathrm{E}-04$ & $8.77 \mathrm{E}-05$ & $1.68 \mathrm{E}-04$ & $7.20 \mathrm{E}-05$ \\
\hline Gamma-chlordane & $1.61 \mathrm{E}-05$ & $6.90 \mathrm{E}-06$ & $1.84 \mathrm{E}-05$ & $7.89 \mathrm{E}-06$ & $1.84 \mathrm{E}-05$ & $7.89 \mathrm{E}-06$ \\
\hline Alpha-endosulfan & $1.84 \mathrm{E}-05$ & $7.89 \mathrm{E}-06$ & $2.53 \mathrm{E}-05$ & $1.08 \mathrm{E}-05$ & - & - \\
\hline $\mathrm{P}, \mathrm{P}^{\prime}-\mathrm{DDE}$ & $2.36 \mathrm{E}-03$ & $1.01 \mathrm{E}-03$ & $1.21 \mathrm{E}-03$ & $5.20 \mathrm{E}-04$ & $1.22 \mathrm{E}-03$ & $5.24 \mathrm{E}-04$ \\
\hline Dieldrin & - & - & - & - & - & - \\
\hline Endrin & - & - & - & - & - & - \\
\hline $\mathrm{P}, \mathrm{P}^{\prime}-\mathrm{DDD}$ & $1.43 \mathrm{E}-04$ & $6.11 \mathrm{E}-05$ & $1.27 \mathrm{E}-04$ & $5.42 \mathrm{E}-05$ & $7.13 \mathrm{E}-05$ & $3.06 \mathrm{E}-05$ \\
\hline $\mathrm{P}, \mathrm{P}^{\prime}-\mathrm{DDT}$ & $4.83 \mathrm{E}-05$ & $2.07 \mathrm{E}-05$ & $4.14 \mathrm{E}-05$ & $1.77 \mathrm{E}-05$ & $3.22 \mathrm{E}-05$ & $1.38 \mathrm{E}-05$ \\
\hline Endosulfan sulfate & $5.06 \mathrm{E}-05$ & $2.17 \mathrm{E}-05$ & $4.14 \mathrm{E}-05$ & $1.77 \mathrm{E}-05$ & $3.68 \mathrm{E}-05$ & $1.58 \mathrm{E}-05$ \\
\hline Methoxychlor & $1.08 \mathrm{E}-04$ & $4.63 \mathrm{E}-05$ & - & - & - & - \\
\hline
\end{tabular}


Table 3. Exposure of SPs pesticide residues in fish for children (Em1) and adults (Em2).

\begin{tabular}{|c|c|c|c|c|c|c|}
\hline Synthetic & Catfish & & Tilapia & & Sardi & \\
\hline pyrethroids & $\begin{array}{c}\text { Em1 } \\
\mathrm{mg} / \mathrm{kg} \cdot \mathrm{d}\end{array}$ & $\begin{array}{c}\mathrm{Em} 2 \\
\mathrm{mg} / \mathrm{kg} \cdot \mathrm{d}\end{array}$ & $\begin{array}{c}\mathrm{Em} 1 \\
\mathrm{mg} / \mathrm{kg} \cdot \mathrm{d}\end{array}$ & $\begin{array}{c}\mathrm{Em} 2 \\
\mathrm{mg} / \mathrm{kg} \cdot \mathrm{d}\end{array}$ & $\begin{array}{c}\mathrm{Em} 1 \\
\mathrm{mg} / \mathrm{kg} \cdot \mathrm{d}\end{array}$ & $\begin{array}{c}\mathrm{Em} 2 \\
\mathrm{mg} / \mathrm{kg} \cdot \mathrm{d}\end{array}$ \\
\hline Cyfluthrin & $7.36 \mathrm{E}-05$ & $3.15 \mathrm{E}-05$ & $6.21 \mathrm{E}-05$ & $2.66 \mathrm{E}-05$ & $2.07 \mathrm{E}-05$ & $8.87 \mathrm{E}-06$ \\
\hline Cypermethrin & - & - & $1.61 \mathrm{E}-05$ & $6.90 \mathrm{E}-06$ & - & - \\
\hline Deltamethrin & - & - & $1.15 \mathrm{E}-05$ & $4.93 \mathrm{E}-06$ & - & - \\
\hline
\end{tabular}

Human health assessment of consumers of pesticide residue contaminated fish was characterized using health risk index (HI). The estimated HIs were obtained by dividing the estimated exposure by its corresponding reference dose by as shown in the Equation (2);

$$
(\mathrm{HI})=\frac{\mathrm{CDI}}{\mathrm{RfD}} \equiv \frac{\mathrm{Em}}{\mathrm{RfD}}
$$

When $\mathrm{HI}$ is less than 1.0 it can be concluded with great certainty that there is essentially no probability of population or community level effect. However, if the ratio exceeds 1.0 then there is a potential for adverse effect of either carcinogenic or non-carcinogenic risk. Carcinogenic risk $\left(\mathrm{CR}_{\circ}\right)$ were estimated using the Equation (3);

$$
\mathrm{CR}_{\circ}=\mathrm{CDI} \times \mathrm{SF} \equiv \mathrm{Em} \times \mathrm{SF}
$$

where Em and SF represents the estimated exposure and slope factor.

For non-carcinogenic risk, the Equation (4);

$$
\text { Risk Quotient }(\mathrm{RQ})=\frac{\text { MEC }}{\text { PNEC }}
$$

where MEC and PNEC represents Measured Environmental Concentration and Predicted No Effect Concentration. The results of RQ calculated values from PEC (MEC) and (PNEC) for the various pesticides residues are shown in Table 4 to estimate risk sediments pose to fish species.

The human health risk assessment of consumers of contaminated fish by pesticides is calculated for children and adults using the Hazard Indices. Table 5 and Table 6 show Hazard Indices of OPs and SPs respectively. There is no certainty of potential adverse effect of cancer or non-cancer effects. Table 7 however show Hazard Index values of OCs with potential risk of cancer or noncancer effects from aldrin and p,p'-DDE for both children and adults from three fish species.

\section{Results and Discussion}

A total of 37 pesticides residues comprising 13 organophosphorus pesticides (OPs) (methamidophos, ethoprophos, phorate, diazinon, fonofos, dimethoate, pirimiphos-methyl, chlorpyrifos, malathion, fenitrothion, parathion, chlorfenvinphos, profenofos), 15 organochlorine pesticides (OCs) $(\beta-\mathrm{HCH}, \gamma-\mathrm{HCH}$, lindane, 
Table 4. RQ estimations from PEC (MEC) and (PNEC).

\begin{tabular}{|c|c|c|c|c|c|c|c|c|c|c|c|}
\hline OPs & PNEC & MEC & RQ & OCs & PNEC & MEC & RQ & SPs & PNEC & MEC & RQ \\
\hline Methamidophos & 0.3 & $\mathrm{BDL}$ & - & Beta-HCH & 1 & 1.207 & 1.207 & Allethrin & 6.85 & 0.202 & 0.0295 \\
\hline Ethoprophos & 0.26 & 0.002 & 0.0077 & Lindane & 0.88 & 0.028 & 0.0318 & Lambda-cyhalothrin & 0.56 & 0.009 & 0.0161 \\
\hline Diazinon & 3 & 0.002 & 0.0007 & Heptachlor & 0 & 0.034 & - & Permethrin & 5 & 0.015 & 0.003 \\
\hline Dimethoate & 1.5 & 0.174 & 0.116 & Aldrin & 0 & 0.19 & - & Cyfluthrin & 0.15 & $\mathrm{BDL}$ & - \\
\hline Pirimiphos-methyl & 16.67 & $\mathrm{BDL}$ & - & Gamma-chlordane & 4.6 & $\mathrm{BDL}$ & - & Cypermethrin & 2.5 & 0.003 & 0.0012 \\
\hline Chlorpyrifos & 1.35 & 0.399 & 0.2956 & Alpha-endosulfan & 0 & $\mathrm{BDL}$ & - & Fenvalerate & 4.5 & 0.004 & 0.0009 \\
\hline Malathion & 21 & 0.272 & 0.0130 & $\mathrm{P}, \mathrm{P}^{\prime}-\mathrm{DDE}$ & 0 & 0.909 & - & Deltamethrin & 1.35 & 0.018 & 0.0133 \\
\hline Fenitrothion & 5.03 & $\mathrm{BDL}$ & - & Dieldrin & 0.65 & 0.009 & 0.0138 & & & & \\
\hline Parathion & 0 & 0.255 & - & Endrin & 0 & 151 & - & & & & \\
\hline Chlorfenvinphos & 0.31 & 0.007 & 0.0226 & $\mathrm{P}, \mathrm{P}^{\prime}-\mathrm{DDD}$ & 0 & 0.457 & - & & & & \\
\hline \multirow[t]{3}{*}{ Profenofos } & 3.58 & $\mathrm{BDL}$ & - & $\mathrm{P}, \mathrm{P}^{\prime}-\mathrm{DDT}$ & 1.13 & 0.189 & 0.1673 & & & & \\
\hline & & & & Endosulfan sulfate & 0.8 & 0.034 & 0.0425 & & & & \\
\hline & & & & Methoxychlor & 60 & 0.005 & $8.33 \mathrm{E}-05$ & & & & \\
\hline
\end{tabular}

Table 5. Health risk assessment of organophosphorus pesticide residues in fish.

\begin{tabular}{|c|c|c|c|c|c|c|c|c|c|c|c|c|}
\hline \multirow[b]{2}{*}{ Pesticides } & \multicolumn{4}{|c|}{ Catfish } & \multicolumn{4}{|c|}{ Tilapia } & \multicolumn{4}{|c|}{ Sardi Fish } \\
\hline & 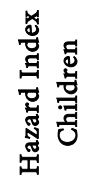 & 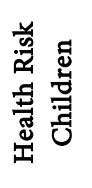 & 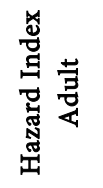 & 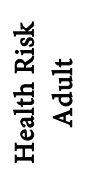 & 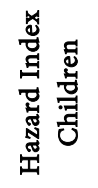 & 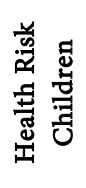 & 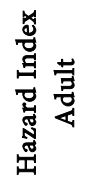 & 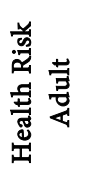 & 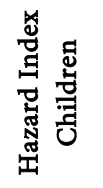 & 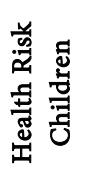 & 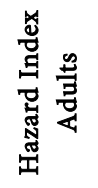 & 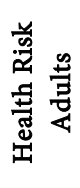 \\
\hline Methamidophos & 0.0610 & NO & 0.0260 & $\mathrm{NO}$ & 0.1090 & NO & 0.0468 & $\mathrm{NO}$ & - & - & - & - \\
\hline Ethoprophos & - & - & - & - & - & - & - & - & - & - & - & - \\
\hline Diazinon & 0.5400 & NO & 0.2320 & NO & 0.2760 & NO & 0.1190 & NO & 0.5200 & NO & 0.2220 & NO \\
\hline Dimethoate & - & - & - & - & 0.2650 & NO & 0.1140 & $\mathrm{NO}$ & - & - & - & - \\
\hline Pirimiphos-methyl & 0.0230 & NO & 0.0099 & $\mathrm{NO}$ & - & - & - & - & - & - & - & - \\
\hline Chlorpyrifos & - & - & - & - & - & - & - & - & - & - & - & - \\
\hline Malathion & 0.0007 & NO & 0.0003 & NO & 0.0015 & NO & 0.0005 & $\mathrm{NO}$ & 0.0006 & NO & 0.0002 & NO \\
\hline Fenitrothion & 0.0037 & NO & 0.0016 & NO & 0.0036 & NO & 0.0016 & NO & 0.0028 & NO & 0.0012 & NO \\
\hline Parathion & - & - & - & - & - & - & - & - & - & - & - & - \\
\hline Chlorfenvinphos & 0.0368 & NO & 0.0158 & NO & 0.0598 & NO & 0.0256 & NO & 0.0322 & NO & 0.0138 & NO \\
\hline Profenofos & 0.2760 & NO & 0.1180 & NO & - & - & - & - & 0.2760 & NO & 0.1180 & NO \\
\hline $\begin{array}{c}\text { Cummulative } \\
\text { risk }\left(\sum \mathrm{HI}\right)\end{array}$ & 0.9412 & & 0.4036 & & 0.4389 & & 0.3075 & & 0.8316 & & 0.3552 & \\
\hline
\end{tabular}

heptachlor, aldrin, $\gamma$-chlordane, p,p'-DDE, p,p'-DDD p,p'-DDT, dieldrin, endrin, $\alpha$-endosulfan, $\beta$-endosulfan, endosulfan sulfate and methoxychlor), and 9 synthetic pyrethroid pesticides (allethrin, bifenthrin, fenpropathrin, $\lambda$-cyhalothrin, permethrin, cyfluthrin, cypermethrin, fenvalerate and deltamethrin) were analyzed in the fish and sediment samples. 
Table 6. Health risk assessment of synthetic pyrethroids pesticide residues in fish.

\begin{tabular}{|c|c|c|c|c|c|c|c|c|c|c|c|c|}
\hline \multirow[b]{2}{*}{ Pesticides } & \multicolumn{4}{|c|}{ Catfish } & \multicolumn{4}{|c|}{ Tilapia } & \multicolumn{4}{|c|}{ Sardi Fish } \\
\hline & 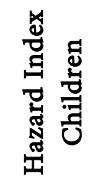 & 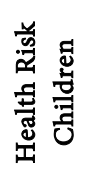 & 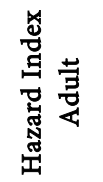 & 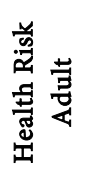 & 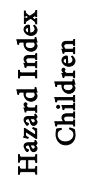 & 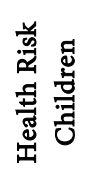 & 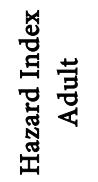 & 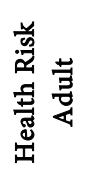 & 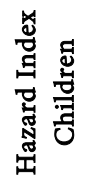 & 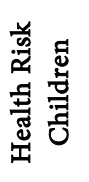 & 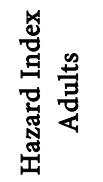 & 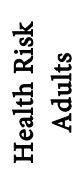 \\
\hline Allethrin & - & NO & - & NO & - & NO & - & NO & - & NO & - & NO \\
\hline Lambda-cyhalothrin & - & NO & - & NO & - & NO & - & NO & - & NO & - & NO \\
\hline Permethrin & - & NO & - & NO & - & NO & - & NO & - & $\mathrm{NO}$ & - & NO \\
\hline Cyfluthrin & 0.0368 & NO & 0.0156 & NO & 0.0311 & NO & 0.0133 & NO & 0.0104 & NO & 0.0044 & NO \\
\hline Cypermethrin & - & NO & - & NO & 0.0081 & NO & 0.0035 & NO & - & NO & - & NO \\
\hline Fenvalerate & 0.0028 & NO & 0.0012 & NO & 0.0005 & NO & 0.0002 & NO & - & NO & - & NO \\
\hline Deltamethrin & - & NO & - & $\mathrm{NO}$ & 0.0012 & $\mathrm{NO}$ & 0.0005 & NO & - & NO & - & NO \\
\hline $\begin{array}{c}\text { Cummulative risk } \\
(\Sigma \mathrm{HI})\end{array}$ & 0.0396 & & 0.0168 & & 0.0409 & & 0.0175 & & 0.0104 & & 0.0044 & \\
\hline
\end{tabular}

Table 7. Health risk assessment of organochlorines pesticide residues in fish.

\begin{tabular}{|c|c|c|c|c|c|c|c|c|c|c|c|c|}
\hline \multirow[b]{2}{*}{ pesticides } & \multicolumn{4}{|c|}{ Catfish } & \multicolumn{4}{|c|}{ Tilapia } & \multicolumn{4}{|c|}{ Sardi Fish } \\
\hline & 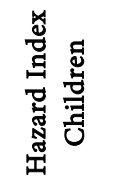 & 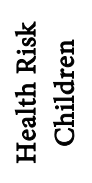 & 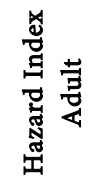 & 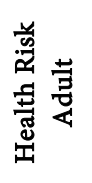 & 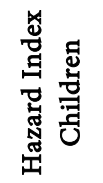 & 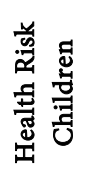 & 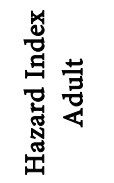 & 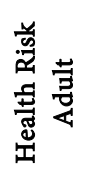 & 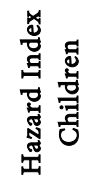 & 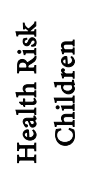 & 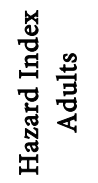 & 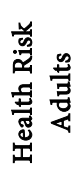 \\
\hline Beta-HCH & - & - & - & - & - & - & - & - & - & - & - & - \\
\hline Lindane & - & - & - & - & - & - & - & - & - & - & - & - \\
\hline Heptachlor & 0.0552 & NO & 0.0236 & NO & 0.0414 & NO & 0.0177 & NO & 0.0552 & NO & 0.0236 & NO \\
\hline Aldrin & $6.8300^{*}$ & YES & $2.9200^{*}$ & YES & $15.87^{*}$ & YES & $6.800^{*}$ & YES & $5.6000^{*}$ & YES & $2.400^{*}$ & YES \\
\hline Gamma-chlordane & 0.0368 & NO & 0.0158 & NO & 0.0322 & NO & 0.0138 & NO & 0.0368 & NO & 0.0158 & NO \\
\hline Alpha-endosulfan & 0.0042 & NO & 0.0018 & NO & 0.0031 & NO & 0.0013 & NO & - & - & - & - \\
\hline $\mathrm{P}, \mathrm{P}^{\prime}-\mathrm{DDE}$ & $2.4200^{\star}$ & YES & $1.0400^{*}$ & YES & $4.720^{*}$ & YES & $2.0200^{\star}$ & YES & $2.4400^{*}$ & YES & $1.050^{*}$ & YES \\
\hline Dieldrin & - & - & - & - & - & - & - & - & - & - & - & - \\
\hline Endrin & - & - & - & - & - & - & - & - & - & - & - & - \\
\hline $\mathrm{P}, \mathrm{P}^{\prime}$-DDT & 0.0828 & NO & 0.0354 & NO & 0.0966 & NO & 0.0414 & NO & 0.0644 & NO & 0.0276 & NO \\
\hline $\mathrm{P}, \mathrm{P}^{\prime}-\mathrm{DDD}$ & 0.0006 & $\mathrm{NO}$ & 0.0003 & NO & 0.0005 & NO & 0.0002 & NO & 0.0003 & NO & 0.0001 & NO \\
\hline Endosulfan sulfate & 0.0069 & NO & 0.0030 & NO & 0.0084 & NO & 0.0084 & NO & 0.0061 & NO & 0.0026 & NO \\
\hline Methoxychlor & - & - & - & - & 0.0216 & NO & 0.0093 & NO & - & - & - & - \\
\hline
\end{tabular}

${ }^{*}$ Values and bold values are hazard indice greater than 1 .

Eight (8) out of the thirteen (13) OPs comprising methamidophos, ethoprophos, diazinon, pirimiphos-methyl, malathion, fenitrothion, chlorfenvinphos and profenofos that were detected in one or more of the samples. Methamidophos and ethoprophos were detected in Catfish and in Tilapia with mean of $0.106 \pm 0.142$ $\mathrm{mg} \cdot \mathrm{kg}^{-1}$ and $0.190 \pm 0.016 \mathrm{mg} \cdot \mathrm{kg}^{-1}$ respectivitly. Ethoprophos was detected only 
in sediment with a mean concentration of $0.002 \pm 0.002 \mathrm{mg} \cdot \mathrm{kg}^{-1}$. Pirimiphosmethyl was detected in both Catfish and in Sediment. Malathion, fenitrothion and chlorfenvinphos were detected above MRLs in all four samples. Profenofos was also detected above MRLs in catfish and sardi fish with mean of $0.006 \pm$ $0.006 \mathrm{mg} \cdot \mathrm{kg}^{-1}$ for both samples.

Residues of fourteen (14) out of the 15 OCs were detected in the samples to be above MRLs. p,p'-DDE recorded the high concentration range of $1.022-1.030$ $\mathrm{mg} / \mathrm{kg}$ in Catfish while $\gamma$-chlordane recorded the lowest concentration range of $0.003-0.10 \mathrm{mg} / \mathrm{kg}$ also in catfish. The mean concentrations of $\beta-\mathrm{HCH}$, dieldrin, endrin and lindane were detected to be above MRLs in sediments. Heptachlor, aldrin, p,p'-DDD, p,p'-DDT, p,p'-DDE and endosulfan-sulfate were detected above MRLs in all fish and sediment samples. Gamma-chlordane was detected in all three fish samples; methoxychlor was detected to be above MRLs in Catfish and in sediments. Beta-HCH recorded the highest concentration of $1.207 \pm 0.351$ $\mathrm{mg} \cdot \mathrm{kg}^{-1}$ in sediment fellowed by $\mathrm{p}, \mathrm{p}^{\prime}$-DDE which was present in all samples at relatively high concentrations. Heptachlor, aldrin, p,p'-DDD, p,p'-DDT and endosulfan sulfate were also detected in all samples. Lindane, dieldrin and endrin were above MRLs in only sediment as $0.028 \pm 0.006 \mathrm{mg} \cdot \mathrm{kg}^{-1}, 0.009 \pm 0.006$ $\mathrm{mg} \cdot \mathrm{kg}^{-1}$ and $0.457 \pm 0.111 \mathrm{mg} \cdot \mathrm{kg}^{-1}$ respectively. Alpha-endosulfan and methoxychlor were both above MRLs in Catfish and Sediment with $0.047 \pm 0.004$ $\mathrm{mg} \cdot \mathrm{kg}^{-1}$ and $0.005 \pm 0.001 \mathrm{mg} \cdot \mathrm{kg}^{-1}$ respectively.

Residues of seven (7) out of the nine (9) synthetic Pyrethroids (SPs) pesticides were detected.

Allethrin, lambda-cyhalothrin and permethrin were detected in sediment at concentrations that were above the MRLs. Their mean concentrations were 0.202 $\pm 0.034 \mathrm{mg} \cdot \mathrm{kg}^{-1}, 0.009 \pm 0.006 \mathrm{mg} \cdot \mathrm{kg}^{-1}$ and $0.015 \pm 0.002 \mathrm{mg} \cdot \mathrm{kg}^{-1}$ respectively. Cyfluthrin was detected at mean concentrations of $0.032 \pm 0.031 \mathrm{mg} \cdot \mathrm{kg}^{-1}, 0.027$ $\pm 0.024 \mathrm{mg} \cdot \mathrm{kg}^{-1}$ and $0.009 \pm 0.004 \mathrm{mg} \cdot \mathrm{kg}^{-1}$ in catfish, tilapia and sardi fish respectively. Fenvalerate was also detected above MRLs in Catfish, Tilapia and in sediment at mean concentrations of $0.024 \pm 0.026 \mathrm{mg} \cdot \mathrm{kg}^{-1}, 0.004 \pm 0.000 \mathrm{mg} \cdot \mathrm{kg}^{-1}$ and $0.004 \pm 0.001 \mathrm{mg} \cdot \mathrm{kg}^{-1}$ respectively. Cypermethrin and deltamethrin were detected in both Tilapia $\left(0.007 \pm 0.004 \mathrm{mg} \cdot \mathrm{kg}^{-1}\right.$ and $\left.0.005 \pm 0.000 \mathrm{mg} \cdot \mathrm{kg}^{-1}\right)$ and Sediment $\left(0.003 \pm 0.002 \mathrm{mg} \cdot \mathrm{kg}^{-1}\right.$ and $\left.0.018 \pm 0.022 \mathrm{mg} \cdot \mathrm{kg}^{-1}\right)$.

\subsection{Ecological Risk Assessment of Fish and Sediment Samples}

The benchmark concentration for carcinogenic effect was derived using USEPA cancer slope factor and the exposure concentration and also for non-carcinogenic effect. Risk assessments were conducted based on the concentrations of OC, OPs and SPs pesticides residues in fish tissues and in sediment. Hazard Indices (HIs) were calculated by dividing the average daily exposure by the reference dose concentrations for fish samples and Risk Quotients were by dividing measured environmental concentrations by predicted no effect concentrations [19]. A hazard ratio which is greater than unity indicates that the average exposure level 
exceeded the benchmark concentration and not acceptable.

From [20], Risk quotient method (RQ) was used to determine the risk sediments pose to aquatic organisms e.g. fish species. RQ is the ratio of the Predicted Environmental Concentration (PEC) to the Predicted No Effect Concentration (PNEC) [21]. The PNEC was estimated by dividing the $\mathrm{LC}_{50}$ with an assessment factor (AF) of 100 [22]. AF is the total uncertainty factor which is from the product of the assumption that the least sensitive humans are 10 times more sensitive than the most sensitive animal species and the additional uncertainty factor of 10 is used to address differences in sensitivity among humans (this is from the assumption that the most sensitive human is 10 times more sensitive than the least sensitive human). This results in a total uncertainty factor of 100 as AF [23]. The results of RQ estimations from PEC (MEC) and (PNEC) [24] the various pesticides residues are calculated.

From [8] and [25] reported that $R Q \geq 1$ indicates a high risk, while $0.1 \leq \mathrm{RQ} \leq$ 1 indicates medium risk, and $0.01 \leq \mathrm{RQ} \leq 0.1$ indicates low risk. Concentrations of pesticide residues in sediment were generally higher than in fish samples. The highest concentration of $\beta$-HCH $\left(1.207 \mathrm{mg} \cdot \mathrm{kg}^{-1}\right)$ was in sediment and second in

p,p'-DDE (1.026 $\left.\mathrm{mg} \cdot \mathrm{kg}^{-1}\right)$. Similar results was obtained for $\mathrm{p}, \mathrm{p}$ '-DDE residue which was predominant in the fate and assessment of persistent organic pollutants in water and sediment [26]. The RQ values of chlorpyrifos (0.0226) and malathion (0.0130) residues were both within the low risk but dimethoate $(0.116)$ and chlorpyrifos $(0.2956)$ were high within the medium risk category. Organochlorines in sediment recorded high concentrations yielding RQ of $\beta-\mathrm{HCH}$ (1.207) which is above the risk and is likely to cause adverse effects to fish species. p,p'-DDT (0.167) indicates medium risk and lindane (0.0318), dieldrin (0.0138) and endosulfan sulfate (0.0425) all indicate low risk levels. RQ values recorded for allethrin (0.0295), lambda-cyhalothrin (0.0161) and deltamethrin (0.0133) were all within the low potential risk level.

\subsection{Carcinogenic Human Risk}

The [27] has defined acceptable risks for carcinogens as within the range $10^{-4}$ to $10^{-6}$ excess lifetime cancer risk. Carcinogenic health risk was calculated for OCs pesticide residues because of their possible cause of cancer to humans. The cancer benchmark concentrations were derived using the oral slope factors (OSFs) of OCs pesticide residues [28]. From [29], individuals have up to a one in between 10,000 to $1,000,000$ chance of not developing cancer in their lifetimes; anything short predisposes humans to cancer risk effects.

Table 8 shows that $\beta$-HCH, lindane and dieldrin did not record any cancer benchmark (CB). p,p'-DDT, p,p'-DDD, $\gamma$-chlordane and heptachlor recorded CB concentrations that were within acceptable cancer risk levels. Aldrin recorded cancer benchmark concentrations that were unacceptable for both children and adults. It was $(8.09 \mathrm{E}-03 ; 3.47 \mathrm{E}-03)$ in Catfish, $(3.49 \mathrm{E}-03 ; 1.49 \mathrm{E}-03)$ in tilapia and $(2.86 \mathrm{E}-03 ; 1.22 \mathrm{E}-03)$ in sardi fish and p,p'-DDE $(8.02 \mathrm{E}-04 \equiv 0.0010)$ in 
Table 8. Cancer benchmark concentrations for organochlorine pesticides residues in fish.

\begin{tabular}{|c|c|c|c|c|c|c|c|}
\hline Organochlorines & $\begin{array}{c}\text { OCs SF } \\
1 /(\mathrm{mg} / \mathrm{kg} / \text { day })\end{array}$ & $\begin{array}{c}\text { Catfish CB } \\
\text { Child }\end{array}$ & $\begin{array}{c}\text { Catfish CB } \\
\text { Adult }\end{array}$ & $\begin{array}{l}\text { Tilapia CB } \\
\text { Child }\end{array}$ & $\begin{array}{c}\text { Tilapia CB } \\
\text { Adult }\end{array}$ & $\begin{array}{l}\text { Sardi Fish } \\
\text { CB Child }\end{array}$ & $\begin{array}{l}\text { Sardi Fish } \\
\text { CB Adult }\end{array}$ \\
\hline Beta-HCH & $1.80 \mathrm{E}+00$ & - & - & - & - & - & - \\
\hline Lindane & $1.30 \mathrm{E}+00$ & - & - & - & - & - & - \\
\hline Heptachlor & $4.50 \mathrm{E}+00$ & $9.32 \mathrm{E}-05$ & $3.99 \mathrm{E}-05$ & $1.24 \mathrm{E}-04$ & $5.31 \mathrm{E}-05$ & $1.24 \mathrm{E}-04$ & $5.31 \mathrm{E}-05$ \\
\hline Aldrin & $1.70 \mathrm{E}+01$ & $8.09 \mathrm{E}-03$ & $3.47 \mathrm{E}-03$ & $3.49 \mathrm{E}-03$ & $1.49 \mathrm{E}-03$ & $2.86 \mathrm{E}-03$ & $1.22 \mathrm{E}-03$ \\
\hline Gamma-chlordane & $3.50 \mathrm{E}-01$ & $5.64 \mathrm{E}-06$ & $2.42 \mathrm{E}-06$ & $6.44 \mathrm{E}-06$ & $2.76 \mathrm{E}-06$ & $6.44 \mathrm{E}-06$ & $2.76 \mathrm{E}-06$ \\
\hline Alpha-endosulfan & NA & - & - & - & - & - & - \\
\hline $\mathrm{P}, \mathrm{P}^{\prime}-\mathrm{DDE}$ & $3.40 \mathrm{E}-01$ & $8.02 \mathrm{E}-04$ & $3.43 \mathrm{E}-04$ & $4.11 \mathrm{E}-04$ & $1.77 \mathrm{E}-04$ & $4.15 \mathrm{E}-04$ & $1.78 \mathrm{E}-04$ \\
\hline Dieldrin & $1.60 \mathrm{E}+01$ & - & - & - & - & - & - \\
\hline Endrin & NA & - & - & - & - & - & - \\
\hline P,P'-DDD & $2.40 \mathrm{E}-01$ & $3.43 \mathrm{E}-05$ & $1.47 \mathrm{E}-05$ & $3.05 \mathrm{E}-05$ & $1.3 \mathrm{E}-05$ & $1.71 \mathrm{E}-05$ & $7.34 \mathrm{E}-06$ \\
\hline P,P'-DDT & $3.40 \mathrm{E}-01$ & $1.64 \mathrm{E}-05$ & $7.04 \mathrm{E}-06$ & $1.41 \mathrm{E}-05$ & $6.02 \mathrm{E}-06$ & $1.09 \mathrm{E}-05$ & $4.69 \mathrm{E}-06$ \\
\hline Endosulfan sulfate & NA & - & - & - & - & - & - \\
\hline Methoxychlor & NA & - & - & - & - & - & - \\
\hline Cumulative CB & & $9.04 \mathrm{E}-03$ & $3.88 \mathrm{E}-03$ & $4.08 \mathrm{E}-03$ & $1.74 \mathrm{E}-03$ & $3.43 \mathrm{E}-03$ & $1.47 \mathrm{E}-03$ \\
\hline
\end{tabular}

Cancer Risk slope factors (OSF) in $1 /\left(\mathrm{mg}_{\mathrm{kg}} \mathrm{d}^{-1}\right)$ were obtained from the USEPA's Integrated Risk Information System (IRIS). ND = Not Available. Values in bold are cancer risk.

children. This indicate a probability of children and adults experiencing carcinogenic effects of aldrin and p,p'-DDE from ingestion of catfish or tilapia or sardi fish in their lifetimes. The cumulative risk posed to humans through fish samples is the sum of total risks from each individual exposure pathway in the type of fish ingested. The cumulative cancer risk of the individual pathways indicates a cancer risk for all of fish species [30].

\subsection{Potential Health Risk Associated with the Consumption of Fish Contaminated with OC, OP and SP Residues from Vea Reservoir}

The Risk Quotient (RQ) values presented in Table 4 are to determine the risk posed to aquatic organisms like fish. $\mathrm{RQ} \geq 1$ indicates a high risk, $0.1 \leq \mathrm{RQ} \leq 1$ indicates a medium risk and $0.01 \leq \mathrm{RQ} \leq 0.1$ indicates a low risk. $\beta$-HCH with $1.207 \mathrm{mg} \cdot \mathrm{kg}^{-1}$ indicates a high risk for organisms in the reservoir.

The Hazard Indices in Tables 5-7 showed that aldrin and p,p'-DDE recorded $\mathrm{HI}>1$. This establishes that there is health risk associated with lifetime consumption of catfish, tilapia and sardi from Vea reservoir. All other pesticides in catfish, tilapia and sardi showed no health hazard associated with their consumption as their Hazard Indices for all the detected residues were $<1$ in spite of their presence in fish. The Oral Slope Factor (OSF) determines the chance of or not developing cancer in the lifetime of a person exposed to contaminated fish. Table 8 showed OSF of OCs where aldrin in catfish, tilapia and in sardi in children and adults and p,p'-DDE in catfish of children with over range bench- 
mark concentrations of developing cancer in their lifetime.

\section{Conclusions and Recommendation}

This research has identified the presence of persistent, bio-accumulative and toxic pesticide residues in fish and sediment at levels that raise public health concerns.

This study has also revealed the presence of $\beta-\mathrm{HCH}$, aldrin and p,p'-DDE in fish and sediment in concentrations above acceptable detectable levels by $\mathrm{WHO} /$ FAO with corresponding non-cancer and cancer risk values. Analysis of health risk assessment exposed systematic toxicity to the ecology and consumers of fish from the Vea reservoir in their lifetime.

Health risk assessment conducted for detected OPs and SPs indicated that these pesticide residues did not pose a non-cancer health threat to children and adults. Cumulative risk assessment of OPs and SPs pesticide mixtures in sampled fish did not also present any health threat.

Non-cancer health risk assessment for OCs pesticides indicated that the estimated Hazard Indices for aldrin and p,p'-DDE were greater than 1 in both children and adults from samples of catfish, tilapia and sardi fish indicating potential adverse human health effects. Carcinogenic risk assessed for OCs pesticide residues indicated that aldrin and p,p'-DDE have cancer benchmark concentrations greater than $10^{-4}$ to $10^{-6}$ threshold for acceptance. Hence there is the possible carcinogenicity in lifetime of consumers of contaminated fish from the Vea reservoir.

An immediate reinforcement of the ban on the use of OCs for irrigation and fishing at the reservoir while pragmatic measures are engaged to stop the consumption of fish from the reservoir is needed by government.

\section{Conflicts of Interest}

The authors declare no conflicts of interest regarding the publication of this paper.

\section{References}

[1] Fianko, J.R., Donkor, A., Lowor, S., Yeboah, P., Glover, E., Adom, T. and Faanu, A. (2011) Health Risk Associated with Pesticide Contamination of Fish from the Densu River Basin in Ghana. Journal of Environmental Protection, 2, 115-123. https://doi.org/10.4236/jep.2011.22013

[2] Rathore, H.S. and Nollet, L.M.L. (2012) Pesticides: Evaluation of Environmental Pollution. CRC Press, Boca Raton. https://doi.org/10.1201/b11864

[3] Darko, G., Osei, A. and Caleb, O. (2008) Persistent Organochlorine Pesticide Residues in Fish, Sediments and Water from Lake Bosomtwi, Ghana. Chemosphere, 72, 21-24. https://doi.org/10.1016/j.chemosphere.2008.02.052

[4] Asante, K.A. and Ntow, W.J. (2009) Status of Environmental Contamination in Ghana, the Perspective of a Research Scientist. In: Obayashi, Y., Isobe, T., Subramanian, A., Suzuki, S. and Tanabe, S., Eds., Interdisciplinary Studies on Environmental Chemistry-Environmental Research in Asia, TERRAPUB, Tokyo, 253-260. 
[5] Bhat, B.A., Bhat, I.A., Vishwakarma, S., Verma, A. and Saxena, G. (2012) A Comparative Study on the Toxicity of a Synthetic Pesticide, Dichlorvos and a Neem Based Pesticide, Neem-On to Labeo rohita (Hamilton). Current World Environment, 7, 157-161. https://doi.org/10.12944/CWE.7.1.24

[6] Willis, G.H. and McDowell, L.L. (1983) Pesticides in Agricultural Runoff and Their Effects on Downstream Water Quality. Environmental Toxicology and Chemistry, 1, 267-279. https://doi.org/10.1002/etc.5620010402

[7] El-Nahhal, Y. and Radwan, A.A. (2013) Human Health Risks: Impact of Pesticide Application. Journal of Environmental and Earth Science, 3, 199-210.

[8] Chitwan, G., Rijal, J.P., Regmi, R., Ghimire, R., et al. (2018) Farmers' Knowledge on Pesticide Safety and Pest Management Practices: A Case Study of Vegetable. Agriculture, 8, 16. https://doi.org/10.3390/agriculture8010016

[9] Dernbach, J.C. and Mintz, J.A. (2011) Environmental Laws and Sustainability: An Introduction. Sustainability, 3, 531-540. https://doi.org/10.3390/su3030531

[10] Dinham, B. (2003) Growing Vegetables in Developing Countries for Local Urban Populations and Export Markets: Problems Confronting Small-Scale Producers. Pest Management Science, 59, 575-582. https://doi.org/10.1002/ps.654

[11] USGS (1999) The Quality of Our Nation's Waters: Nutrients and Pesticides-A Summary. Science for a Changing World, 1-4.

[12] USEPA (2004) Overview of the Ecological Risk Assessment Process in the Office of Pesticide Programs, Office of Pesticide Programs.

[13] Lartey, A. (2017) Seafood as Part of a Healthy Diet.

[14] Kafilzadeh, F. (2015) Assessment of Organochlorine Pesticide Residues in Water, Sediments and Fish from Lake Tashk, Iran. Achievements in the Life Sciences, 9, 107-111.

[15] Pelig-Ba, K.B. (2011) Levels of Agricultural Pesticides in Sediments and Irrigation Water from Tono and Vea in the Upper East of Ghana. Journal of Environmental Protection, 2, 761-768. https://doi.org/10.4236/jep.2011.26088

[16] Sun, P.C., Tsai, R.J., Finger, G., Chen, Y.Y. and Yeh, D. (2008) What Drives a Successful E-Learning? An Empirical Investigation of the Critical Factors Influencing Learner Satisfaction. Computers \& Education, 50, 1183-1202. https://doi.org/10.1016/j.compedu.2006.11.007

[17] Ahmad, D. and Afzal, M. (2020) Flood Hazards and Factors Influencing Household Flood Perception and Mitigation Strategies in Pakistan. Environmental Science and Pollution Research International, 27, 15375-15387. https://doi.org/10.1007/s11356-020-08057-z

[18] Boobis, A.R., Cohen, S.M., Dellarco, V., Mcgregor, D., Meek, M.E.B., Vickers, C., et al. (2006) IPCS Framework for Analyzing the Relevance of a Cancer Mode of Action for Humans on Chemical Safety in Developing the Framework for Analysing the Relevance. Critical Reviews in Toxicology, 36, 781-792. https://doi.org/10.1080/10408440600977677

[19] Ilboudo, S., Toé, A.M., Ouédraogo, R., Ouédraogo, M. and Guissou, I.P. (2014) Ecological Risk Assessment of Pesticide Residues in Water from Desert Locust Area in Burkina Faso Ecological Risk Assessment of Pesticide Residues in Water from Desert Locust Area in Burkina Faso. Research Journal of Environmental and Earth Sciences, 4, 227-232. https://doi.org/10.19026/rjees.6.5764

[20] ECHA (2013) Principles for Environmental Risk Assessment of the Sediment Compartment. Proceedings of the Topical Scientific Workshop, Helsinki, 7-8 May, 2013, 
$82 \mathrm{p}$.

[21] Lawrence, E., Ozekeke, O. and Isioma, T. (2015) Distribution and Ecological Risk Assessment of Pesticide Residues in Surface Water, Sediment and Fish from Ogbesse River, Edo State, Nigeria. Journal of Environmental Chemistry and Ecotoxicology, 7, 20-30.

[22] Maund, S.J., Sherratt, T.N., Stickland, T., Williams, P., Shillabeer, N. and Jepson, P.C. (1997) Ecological Considerations in Pesticide Risk Assessment for Aquatic Ecosystems. Pesticide Science, 49, 185-190.

https://doi.org/10.1002/(SICI)1096-9063(199702)49:2<185::AID-PS493>3.0.CO;2-M

[23] Lamers, M., Schreinemachers, P., Ingwersen, J., Sangchan, W., Grovermann, C. and Berger, T. (2013) Agricultural Pesticide Use in Mountainous Areas of Thailand and Vietnam: Towards Reducing Exposure and Rationalizing Use. In: Fröhlich, H.L., Schreinemachers, P., Stahr, K. and Clemens, G., Eds., Sustainable Land Use and Rural Development in Southeast Asia: Innovations and Policies for Mountainous Areas, Springer, Berlin, Heidelberg, 149-173.

https://doi.org/10.1007/978-3-642-33377-4_4

[24] ECETOC (2004) Soil and Sediment Assessment of Organic Chemicals, No. 92.

[25] Sanchez-Bayo, F. (2002) An Approach for Ecological Risk Assessment of Pesticides in Agriculture. Journal of Pesticide Science, 27, 425-428.

https://doi.org/10.1584/jpestics.27.425

[26] Zhang, Z.L. (2003) Fate and Assessment of Persistent Organic Pollutants in Water and Sediment from Minjiang River Estuary, Southeast China. Chemosphere, 52, 1423-1430. https://doi.org/10.1016/S0045-6535(03)00478-8

[27] US EPA (2014) Framework for Human Health Risk Assessment to Inform Decision Making. Framework for Human Health Risk Assessment to Inform Decision Making, 1-63.

[28] Saria, J.A.M. (2017) Assessment of Human Health Hazard Due to Metal Uptake via Fish Consumption from Coastal Area of Tanzania. Ethiopian Journal of Environmental Studies and Management, 10, 555-565.

https://doi.org/10.4314/ejesm.v10i4.12

[29] U. EPA (1992) Guidelines for Exposure Assessment.

[30] Sax, S.N., Bennett, D.H., Chillrud, S.N., Ross, J., Kinney, P.L. and Spengler, J.D. (2006) A Cancer Risk Assessment of Inner-City Teenagers Living in New York City and Los Angeles. Environmental Health Perspectives, 114, 1558-1566.

https://doi.org/10.1289/ehp.8507 